\title{
High-Fidelity Preparation, Gates, Memory, and Readout of a Trapped-Ion Quantum Bit
}

\author{
T. P. Harty, ${ }^{1}$ D. T. C. Allcock, ${ }^{1}$ C. J. Ballance, ${ }^{1}$ L. Guidoni, ${ }^{1,2}$ H. A. Janacek, ${ }^{1}$ \\ N. M. Linke, ${ }^{1}$ D. N. Stacey, ${ }^{1}$ and D. M. Lucas ${ }^{1, *}$ \\ ${ }^{1}$ Clarendon Laboratory, Department of Physics, University of Oxford, Parks Road, Oxford OX1 3PU, United Kingdom \\ ${ }^{2}$ Laboratoire Matériaux et Phénomènes Quantiques, University of Paris Diderot, UMR 7162 CNRS, F-75205 Paris, France
} (Received 19 May 2014; revised manuscript received 2 September 2014; published 24 November 2014)

\begin{abstract}
We implement all single-qubit operations with fidelities significantly above the minimum threshold required for fault-tolerant quantum computing, using a trapped-ion qubit stored in hyperfine "atomic clock" states of ${ }^{43} \mathrm{Ca}^{+}$. We measure a combined qubit state preparation and single-shot readout fidelity of $99.93 \%$, a memory coherence time of $T_{2}^{*}=50 \mathrm{sec}$, and an average single-qubit gate fidelity of $99.9999 \%$. These results are achieved in a room-temperature microfabricated surface trap, without the use of magnetic field shielding or dynamic decoupling techniques to overcome technical noise.
\end{abstract}

DOI: 10.1103/PhysRevLett.113.220501

PACS numbers: 03.67.Lx, 37.10.Ty

The great potential of quantum computing requires two essential ingredients for its realization: high-fidelity quantum logic operations and a physical implementation which can be scaled up to large numbers of quantum bits [1]. Among the candidate technologies for implementing quantum information processing, individual trapped ions were recognized early as a very promising system [2-4]: the qubits are stored in internal atomic energy levels of the ions, which can be extremely stable and well isolated from the environment, and the strong Coulomb interaction between neighboring ions can be used to mediate qubit-qubit logic. Since the first proposals, multiple-qubit algorithms have been demonstrated [5], and there has been significant progress in developing scalable ion trap technologies [6]. Long qubit memory coherence time [7], high-fidelity state preparation and readout [8], and single-qubit gates with fault-tolerant error rates [9] have all been demonstrated, in a variety of different trapped ions and experiments.

In this Letter, we demonstrate all single-qubit operations (preparation, memory, gates, and readout) with performances comparable to or better than previous work, and all in the same system. All errors are more than an order of magnitude below the $\approx 1 \%$ fault-tolerant thresholds emerging from recent numerical calculations using surface-code error correction [10]; this is critical for the practical implementation of fault-tolerant methods, whose resource requirements increase dramatically for error rates close to threshold [11]. Furthermore, the ion qubit is trapped in a microfabricated surface-electrode trap [12] with a twodimensional electrode layout which is extendable to large arrays of multiplexed traps, as envisaged in the original proposal for scalable trapped-ion quantum information processing [4]. Below, we describe the trap and the ${ }^{43} \mathrm{Ca}^{+}$qubit, along with three experiments performed to measure the combined state preparation and readout error, the qubit coherence time, and the average single-qubit gate error.
The ion trap is of a novel design which incorporates integrated microwave (m.w.) circuitry (resonators, waveguides, and coupling elements) designed to allow single- and two-qubit quantum logic gates to be driven by near-field microwaves $[13,14]$ instead of by lasers: this will enable all the coherent qubit operations to be performed by electronic techniques, where one can take advantage of readily available microwave sources whose power and absolute frequency are very stable, and which can be easily connected to the trap electrodes. In contrast to solid-state qubit technologies [15], it is not necessary to cool the apparatus to milli-Kelvin temperatures, as the microwave control fields are classical: only the qubits themselves need to be cold, and this is straightforwardly achieved using Doppler laser cooling. A schematic diagram of the trap and the laser beam layout is shown in Fig. 1(a); the trap is described in more detail in Ref. [16].

A single ${ }^{43} \mathrm{Ca}^{+}$ion is loaded into the trap from a $12 \%$ enriched calcium source using isotope-selective photoionization [17]. Trap secular frequencies are typically $3 \mathrm{MHz}$ (radial) and $500 \mathrm{kHz}$ (axial). The ion is Doppler cooled with lasers operating at 397 and $866 \mathrm{~nm}$; further lasers at 393, 850, and $854 \mathrm{~nm}$ are used for qubit readout and reset. An advantage of the $\mathrm{Ca}^{+}$ion is that all wavelengths are available from solid-state diode lasers without the need for frequency doubling, are compatible with integrated optics [18], and do not cause observable charging of the trap structure under normal operation. The optical operations (cooling, state preparation, and readout) are robust to laser intensity and frequency noise (laser linewidths are $\approx 1 \mathrm{MHz}$ ), and only require lowpower beams.

Hyperfine states in the ground $4 S_{1 / 2}$ level of the ion are used for the qubit states [Fig. 1(b)]. As spontaneous decay rates are negligible, these states have essentially infinite $T_{1}$ times (limited in practice by the ion trapping lifetime, which is typically several hours in this trap under ultrahigh 
(a)

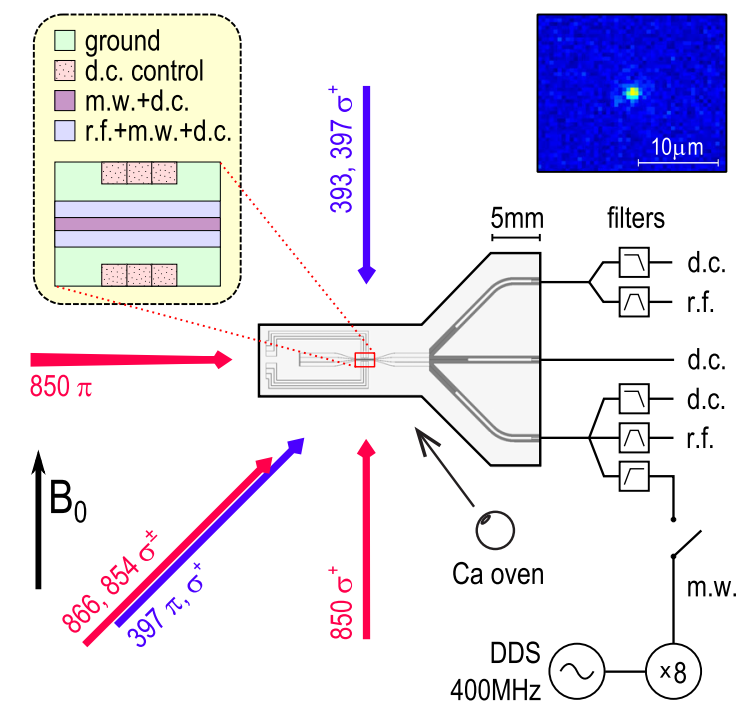

(b)

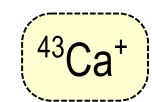

$3 \mathrm{D}_{5}$

12

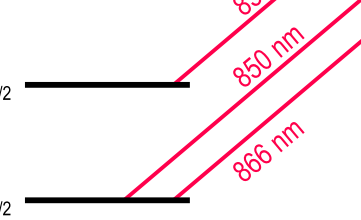

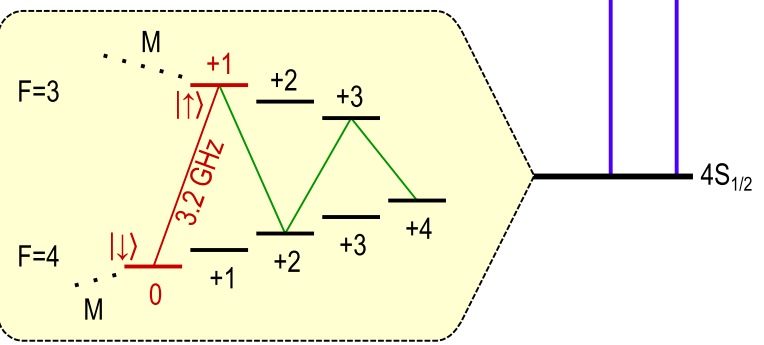

FIG. 1 (color online). The ion trap and the qubit. (a) Schematic diagram of the surface ion trap, showing (left-hand inset) central electrode layout. Microwave (m.w., $3.2 \mathrm{GHz}$ ) signals are combined with the trap radio frequency voltage (rf, $40 \mathrm{MHz}$ ) via filters, as indicated here for the lower axial electrode. Also shown are laser beam directions and polarizations with respect to the static magnetic field $B_{0}=146 \mathrm{G}$. The violet $397 \mathrm{~nm}$ Doppler-cooling beam is elliptically polarized such that it contains only $\pi$ and $\sigma^{+}$polarizations, and copropagates with linearly $\left(\sigma^{ \pm}\right)$polarized infrared repumping beams. Circularly $\sigma^{+}$and linearly $\pi$ polarized beams are used for state preparation and readout (the $\pi$ beam is at $\sim 45^{\circ}$ to the plane of the figure and reflects off the trap surface). To load the trap, neutral $\mathrm{Ca}$ atoms effusing from the oven are ionized by laser beams at 423 and $389 \mathrm{~nm}$ which copropagate with the Doppler-cooling beams. Ion fluorescence is collected by an imaging system perpendicular to the plane of the trap; the right-hand inset shows an image of a single ${ }^{43} \mathrm{Ca}^{+}$ion, which is trapped $75 \mu \mathrm{m}$ above the electrodes. (b) ${ }^{43} \mathrm{Ca}^{+}$level structure, showing optical transitions used for Doppler cooling, qubit state preparation, and readout. The inset shows part of the ground level hyperfine structure, labeled by quantum numbers $F$ and $M$, with the qubit $|\downarrow\rangle$ and $|\uparrow\rangle$ states, the $3.2 \mathrm{GHz}$ qubit transition (red), and the auxiliary transitions (green) used for state preparation and readout. The Zeeman splittings between adjacent $M$ states are $\approx 50 \mathrm{MHz}$.

vacuum conditions, $<10^{-11}$ torr). $T_{2}$ coherence times are limited by the frequency stability of the qubit transition. The state energies depend on the static magnetic field $B$ through the Zeeman effect, and ambient magnetic field noise would normally limit the coherence time to a few milliseconds. However, certain transition energies become independent of magnetic field to first order at particular values of the field, due to the nonlinear dependence arising from hyperfine state mixing, and these permit particularly stable qubits [7]. We choose one of these so-called "atomic clock" transitions, $S_{1 / 2}^{4,0} \leftrightarrow S_{1 / 2}^{3,+1}$ (where the superscripts denote angular momentum quantum numbers $F, M$ ), which in ${ }^{43} \mathrm{Ca}^{+}$is field independent at $B_{0} \approx 146 \mathrm{G}$ [Fig. 2(a)]. Unlike schemes for microwave quantum logic based on static magnetic field gradients [19], the use of near-field microwaves allows all qubits to share the same, welldefined, noise-immune, frequency.

The relatively large magnetic field leads to a complex atomic level structure, with Zeeman splittings spanning $\sim 500 \mathrm{MHz}$, and because of the low-lying $D$ levels in $\mathrm{Ca}^{+}$ there is no closed cycling transition for laser cooling. We have nevertheless identified a simple Doppler cooling method which requires only two $397 \mathrm{~nm}$ frequencies, a single $866 \mathrm{~nm}$ frequency, moderate laser powers $(\sim 100 \mu \mathrm{W})$, and a single beam direction. We obtain a fluorescence count rate comparable to that from a single, saturated, ${ }^{40} \mathrm{Ca}^{+}$ion, at $50000 \mathrm{~s}^{-1}$ with a net photon detection efficiency of $0.3 \%$, which is sufficient for high-fidelity fluorescence detection.

To measure the combined state preparation and measurement (SPAM) error, we repeatedly prepare the same qubit state, and read it out, averaging over preparations of the $|\downarrow\rangle$ and $|\uparrow\rangle$ states. We first optically pump the ion to the $S_{1 / 2}^{4,+4}$ state using circularly $\sigma^{+}$polarized $397 \mathrm{~nm}$ light. We use a microwave technique to improve the optical pumping fidelity, which is otherwise limited by imperfect polarization of the $397 \mathrm{~nm}$ beam (see Supplemental Material [22]). A series of three (or four) microwave $\pi$ pulses on the transitions indicated in the inset of Fig. 1(b) then transfers the ion to the $|\uparrow\rangle$ (or $|\downarrow\rangle$ ) qubit state, as desired. To read out the qubit state, three microwave $\pi$ pulses transfer population in $|\uparrow\rangle$ to $S_{1 / 2}^{4,+4}$, and a fourth $\pi$ pulse transfers $|\downarrow\rangle \rightarrow S_{1 / 2}^{3,+1}$. Population in $S_{1 / 2}^{4,+4}$ is then "shelved" in the metastable $3 D_{5 / 2}$ level by a 

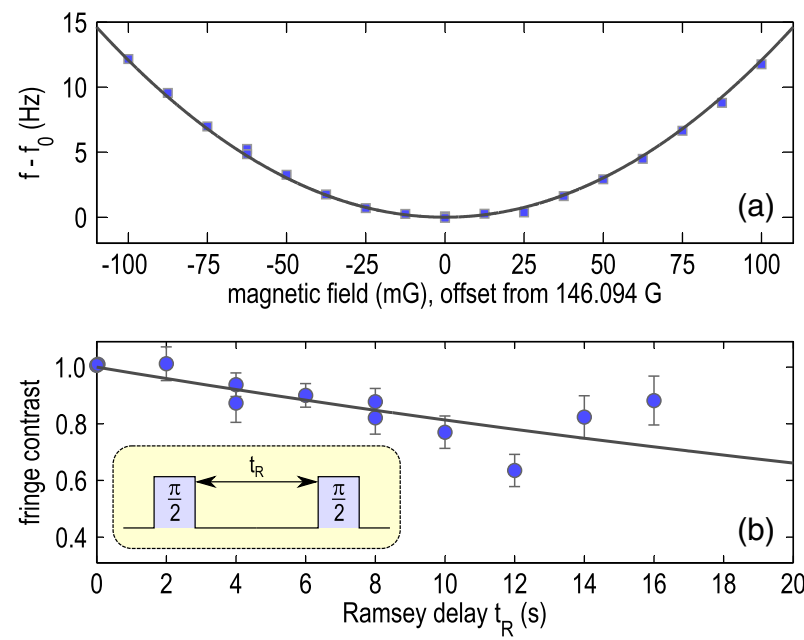

FIG. 2 (color online). (a) Microwave spectroscopy of the qubit transition, varying the static magnetic field $B$ through the fieldindependent point $B_{0}=146.094 \mathrm{G}$. At each field value, the qubit transition frequency $f$ was measured by Ramsey spectroscopy, to a precision $\approx 0.1 \mathrm{~Hz}$. The field-independent qubit transition is at $f_{0}=3199941077 \mathrm{~Hz}$ after adjusting for a $-5 \mathrm{~Hz}$ shift due to the trap (see text). The solid line shows the expected frequency calculated using the Breit-Rabi formula assuming the known zero-field hyperfine splitting [20] and a nuclear magnetic moment [21] of $\mu_{I}=-1.31535 \mu_{N}$. (b) Qubit coherence time measurements. At each value of the Ramsey free precession time $t_{R}$ the phase of the second $\pi / 2$ pulse was varied to produce a set of Ramsey fringes. The contrast of the fringes is fitted with an exponential decay, giving a coherence time $T_{2}^{*}=50(10) \mathrm{sec}$.

repeated sequence of $\left(393 \mathrm{~nm} \sigma^{+}, 850 \mathrm{~nm} \sigma^{+}, 850 \mathrm{~nm} \pi\right.$ polarized) optical pumping pulses, as described in Ref. [8]. Finally, the Doppler-cooling lasers are applied again and we detect whether or not the ion was shelved by the absence or presence of $397 \mathrm{~nm}$ fluorescence.

For 150000 preparations of each qubit state, we measure the combined SPAM error to be 6.8(5) $\times 10^{-4}$ (Fig. 3). As the qubit readout method is not a quantum nondemolition measurement, we cannot repeat it many times to separate the preparation and readout errors, but from estimates of the various contributions to the combined error (Table I), we assign errors of $\approx 2 \times 10^{-4}$ to the state preparation and $\approx 5 \times 10^{-4}$ to the readout. The error contributions could all be reduced by technical improvements (e.g., increasing the photon detection efficiency [8]), except for that due to the shelving transfer to $D_{5 / 2}$, which is limited to a minimum [21] of $\approx 1 \times 10^{-4}$ (at $B_{0}=146 \mathrm{G}$ ) by the atomic structure of ${ }^{43} \mathrm{Ca}^{+}$.

The qubit coherence time was measured by performing Ramsey experiments (without any dynamic decoupling pulses [24]) on the $S_{1 / 2}^{4,0} \leftrightarrow S_{1 / 2}^{3,+1}$ qubit transition at $f_{0} \approx$ $3.200 \mathrm{GHz}$. To ensure that the applied magnetic field remained close to the field-independent point, the frequency of the field-dependent $S_{1 / 2}^{4,+4} \leftrightarrow S_{1 / 2}^{3,+3}$ transition was periodically measured by the computer controlling the

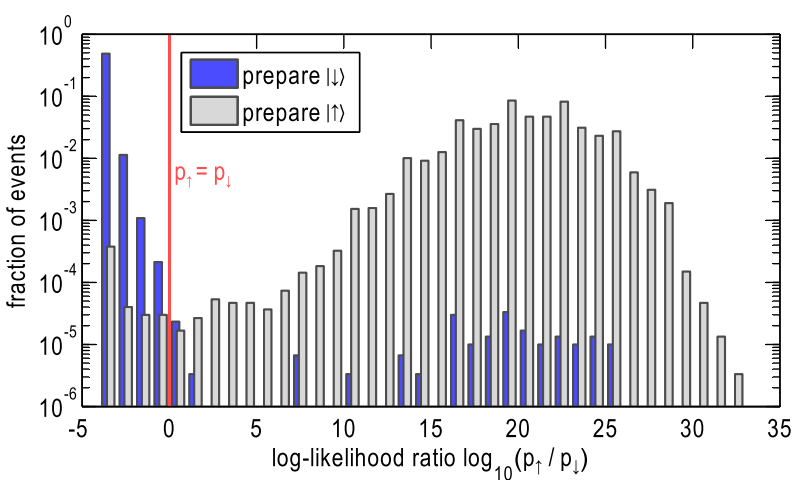

FIG. 3 (color online). Qubit state preparation and measurement (SPAM) results. We prepared and measured the $|\downarrow\rangle$ (blue histogram) and $|\uparrow\rangle$ (gray histogram) qubit states 150000 times each. For each measurement the likelihood $p_{\downarrow}$ (or $p_{\uparrow}$ ) that the state was $|\downarrow\rangle$ (or $|\uparrow\rangle$ ) was calculated from the time-resolved photon counts detected on a photomultiplier (see Ref. [8]); if $p_{\uparrow}>p_{\downarrow}$, we infer that the qubit was in the $|\uparrow\rangle$ state, and vice versa. If the state inferred disagrees with the state prepared, either a preparation or a measurement error has occurred; thus, the fraction of experiments in which an error occurred is given by the sum of the blue events above the $p_{\uparrow}=p_{\downarrow}$ threshold $\left(2 \times 10^{-4}\right)$ and the gray events below threshold $\left(5 \times 10^{-4}\right)$.

experiment, and an appropriate correction was applied to the magnetic field coil current. Ramsey delays up to $t_{R}=16 \mathrm{sec}$ were used, with results shown in Fig. 2(b). An exponential decay $\exp \left(-t_{R} / T_{2}^{*}\right)$ fitted to the data gives a coherence time $T_{2}^{*}=50(10) \mathrm{sec}$. The coherence time may be limited by residual magnetic field drift (the qubit's second-order field dependence is $d^{2} f / d B^{2}=$ $2.4 \mathrm{mHz} / \mathrm{mG}^{2}$ ), instability of the local oscillator, and fluctuations in the amplitude of the trap rf voltage (by varying the rf power and extrapolating to zero power, we

TABLE I. Error contributions: (top) state preparation and readout experiment, (bottom) single-qubit randomized benchmarking experiment (EPG: error per gate). The error contributions are estimates based on auxiliary experiments, experimentally measured parameters, and theoretical models of the various processes (see Supplemental Material [22] and Ref. [23]).

\begin{tabular}{lc}
\hline \hline Preparation or readout operation & Error \\
\hline Stretched state $S_{1 / 2}^{4,+4}$ preparation & $<1 \times 10^{-4}$ \\
Transfer to qubit $(3$ or 4 m.w. $\pi$ pulses) & $1.8 \times 10^{-4}$ \\
Transfer from qubit $(4 \mathrm{~m} . w . \pi$ pulses) & $1.8 \times 10^{-4}$ \\
Shelving transfer $S_{1 / 2}^{4,+4} D_{5 / 2}$ & $1.7 \times 10^{-4}$ \\
Time-resolved fluorescence detection & $1.5 \times 10^{-4}$ \\
\hline \hline Single-qubit gate error source & Mean EPG \\
\hline Microwave detuning offset $(4.5 \mathrm{~Hz})$ & $0.7 \times 10^{-6}$ \\
Microwave pulse area error $\left(5 \times 10^{-4}\right)$ & $0.3 \times 10^{-6}$ \\
Off-resonant effects & $0.1 \times 10^{-6}$ \\
\hline \hline
\end{tabular}


measure a differential ac Zeeman shift of $-5 \mathrm{~Hz}$ due to $\mathrm{rf}$ currents in the trap electrodes [25]). The reduction in fringe contrast could also be due to effects unrelated to the qubit coherence, for example, heating of the ion during $t_{R}$, which increases readout error due to Doppler broadening of the $393 \mathrm{~nm}$ shelving transition. We note that longer coherence times have been measured in large ensembles, using trapped ions [26] and nuclear spins [27] (in the latter case, only with multiple dynamical decoupling pulses).

The fidelity of single-qubit gates driven by one of the near-field integrated microwave electrodes was measured by the established technique of randomized benchmarking [28], which yields an average gate error appropriate to a computational context. We use the same method as Ref. [9], which reports the previous lowest single-qubit gate error. Having prepared the qubit in $|\uparrow\rangle$, we apply a preprogrammed pseudorandom sequence of logical gates, where each logical gate comprises a Pauli gate followed by a Clifford gate. The sequence terminates by rotating the qubit into either $|\downarrow\rangle$ or $|\uparrow\rangle$, chosen with equal probability. Clifford gates are randomly chosen to rotate the qubit about the $\pm x$ or $\pm y$ axes on the Bloch sphere; Pauli gates are randomly chosen to rotate about the $\pm x, \pm y$, or $\pm z$ axes, or to be a $\pm I$ identity gate. In the experiment, each Clifford gate is performed by a microwave $\pi / 2$ pulse and each Pauli gate by a pair of $\pi / 2$ pulses. Identity gates are implemented using delays of the same duration (12 $\mu \mathrm{s})$ as the $\pi / 2$ pulses, $\pm z$ rotations as an identity followed by a rotation of the logical frame of the qubit for subsequent pulses. The microwaves are generated by a frequencyoctupled $400 \mathrm{MHz}$ direct digital synthesis (DDS) source, fed via a switch to one of the m.w. electrodes [Fig. 1(a)]; the enhancement provided by the integrated m.w. resonator and the proximity of the ion to the electrode means that a low m.w. power $(0.1 \mathrm{~mW})$ is sufficient and a power amplifier is not necessary. The m.w. power was periodically calibrated during the experiments using a sequence of 751 $\pi / 2$ pulses. The qubit was kept at the field-independent point by servoing the magnetic field as in the coherence time measurements.

Each pseudorandom sequence is applied many times, and we compare the measured final qubit state with the expected outcome for that sequence. We apply sequences of various lengths, up to 2000 computational gates, and use 32 distinct sequences at each length, for a total of 224 randomly chosen sequences. Since some sequences are more susceptible to errors than others, we performed numerical simulations to check that this provided sufficient randomization over systematic variations (see Supplemental Material [22]). Experimental results are shown in Fig. 4, where we deduce an average error per gate of $1.0(3) \times 10^{-6}$ from the slope of the fitted line. This is an upper limit since it ignores any possible increase in SPAM error for the longer sequences; an independent experiment comparing 2000-gate runs with control runs that contained no gates, but had the same
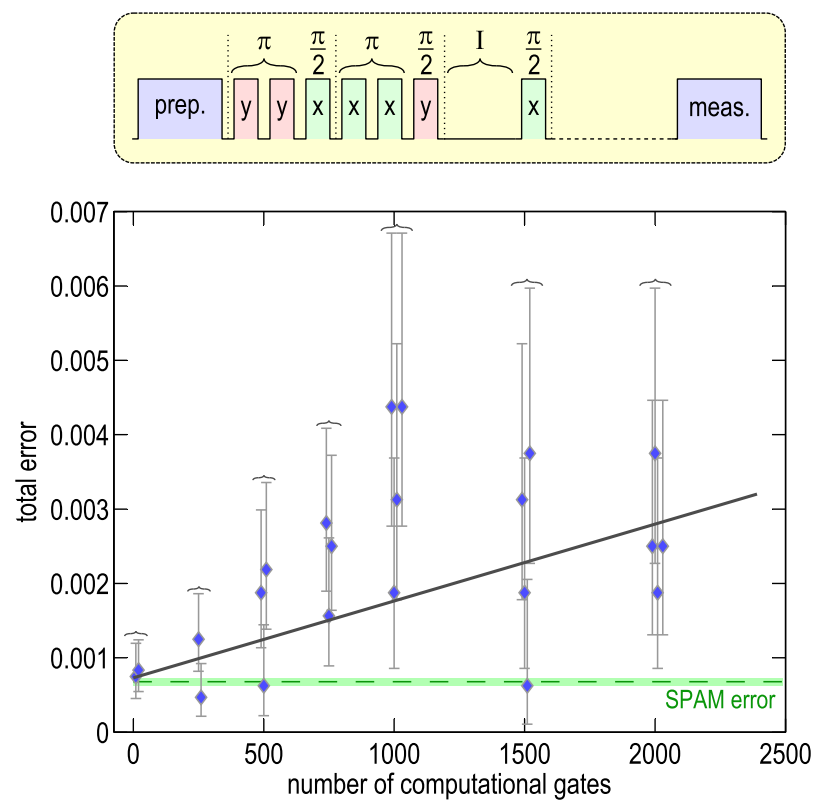

FIG. 4 (color online). Randomized benchmarking of singlequbit gates. The start of an example pseudorandom sequence is shown: each computational gate is composed of three physical $\pi / 2$ pulses (absent in the case of identity $I$ operations or $z$ rotations). Additional $\pi / 2$ pulses at the end of the sequence rotate the qubit into the $(|\downarrow\rangle,|\uparrow\rangle)$ basis for measurement. If the measured state disagrees with that expected, an error is recorded. For each sequence length, 32 distinct sequences are used, each one being repeated the same number of times (typically 100) to measure the total error. Results are shown in the plot, where repeated runs have been offset horizontally for clarity. The gradient of a weighted straight line fit gives an average error per gate of $1.0(3) \times 10^{-6}$, while the intercept agrees with the independently measured SPAM error of $6.8(5) \times 10^{-4}$ (dashed line). Error bars represent $68 \%$ confidence intervals, assuming binomial statistics. The fit gives a reduced $\chi^{2} \approx 0.84$.

160 ms delay between preparation and readout, gave an error per gate of $0.4(8) \times 10^{-6}$. Estimated contributions to the measured gate error are shown in Table I; these can all be reduced or compensated for by technical improvements (for example, a trap design allowing arbitrary control of the microwave polarization [29] could eliminate the off-resonant excitation of other m.w. transitions).

In conclusion, we have used a new magnetic-fieldindependent qubit in ${ }^{43} \mathrm{Ca}^{+}$, held in a scalable ion trap design, to demonstrate all single-qubit operations at error rates more than an order of magnitude below the threshold necessary for surface-code quantum error correction. The coherence time and gate fidelity surpass measurements in all other single physical qubits. The combined state preparation and readout error is the lowest measured for any atomic clock qubit. Although we did not need to employ composite pulse techniques to correct for technical noise, the exceedingly low single-qubit gate error means that the extensive library of such techniques [30] is usable with negligible error overhead. In separate experiments 
[31], we have demonstrated laser-driven two-qubit quantum logic gates on ${ }^{43} \mathrm{Ca}^{+}$hyperfine qubits with a fidelity $>99 \%$, showing that all quantum logic operations can be performed using this ion with a precision at or above the current state of the art $[32,33]$.

It is a pleasure to thank E. Knill and A. Meier for advice regarding the randomized benchmarking experiment, A. Steane and J. Jones for helpful discussions, and J. Home for comments on the manuscript. This work was supported by the EPSRC.

*d.lucas@physics.ox.ac.uk

[1] M. A. Nielsen and I. L. Chuang, Quantum Computation and Quantum Information (Cambridge University Press, Cambridge, England, 2000).

[2] J. I. Cirac and P. Zoller, Phys. Rev. Lett. 74, 4091 (1995).

[3] A. Steane, Appl. Phys. B 64, 623 (1997).

[4] D. J. Wineland, C. Monroe, W. M. Itano, D. Leibfried, B. E. King, and D. M. Meekhof, J. Res. NIST 103, 259 (1998).

[5] R. Blatt and D. Wineland, Nature (London) 453, 1008 (2008).

[6] C. Monroe and J. Kim, Science 339, 1164 (2013).

[7] C. Langer et al., Phys. Rev. Lett. 95, 060502 (2005).

[8] A. H. Myerson, D. J. Szwer, S. C. Webster, D. T. C. Allcock, M. J. Curtis, G. Imreh, J. A. Sherman, D. N. Stacey, A. M. Steane, and D. M. Lucas, Phys. Rev. Lett. 100, 200502 (2008).

[9] K. R. Brown, A. C. Wilson, Y. Colombe, C. Ospelkaus, A. M. Meier, E. Knill, D. Leibfried, and D. J. Wineland, Phys. Rev. A 84, 030303 (2011).

[10] A. G. Fowler, M. Mariantoni, J. M. Martinis, and A. N. Cleland, Phys. Rev. A 86, 032324 (2012).

[11] A. M. Steane, Phys. Rev. A 68, 042322 (2003).

[12] S. Seidelin et al., Phys. Rev. Lett. 96, 253003 (2006).

[13] C. Ospelkaus, U. Warring, Y. Colombe, K. R. Brown, J. M. Amini, D. Leibfried, and D. J. Wineland, Nature (London) 476, 181 (2011).

[14] U. Warring, C. Ospelkaus, Y. Colombe, R. Jördens, D. Leibfried, and D. J. Wineland, Phys. Rev. Lett. 110, 173002 (2013).
[15] M. H. Devoret and R. J. Schoelkopf, Science 339, 1169 (2013)

[16] D. T. C. Allcock, T. P. Harty, C. J. Ballance, B. C. Keitch, N. M. Linke, D. N. Stacey, and D. M. Lucas, Appl. Phys. Lett. 102, 044103 (2013).

[17] D. M. Lucas, A. Ramos, J. P. Home, M. J. McDonnell, S. Nakayama, J.-P. Stacey, S. C. Webster, D. N. Stacey, and A. M. Steane, Phys. Rev. A 69, 012711 (2004).

[18] G. R. Brady et al., Appl. Phys. B 103, 801 (2011).

[19] F. Mintert and C. Wunderlich, Phys. Rev. Lett. 87, 257904 (2001); 91, 029902 (2003).

[20] F. Arbes, M. Benzing, Th. Gudjons, F. Kurth, and G. Werth, Z. Phys. D 31, 27 (1994).

[21] T. P. Harty, Ph.D. thesis, University of Oxford, 2013, http://ora.ox.ac.uk/objects/uuid:55264c2d-bb42-4439-bf49 $-731 \mathrm{~b} 9 \mathrm{f} 66 \mathrm{de} 74$.

[22] See Supplemental Material at http://link.aps.org/ supplemental/10.1103/PhysRevLett.113.220501 for further experimental details and the basis for the error contributions given in Table I.

[23] D. J. Szwer, Ph.D. thesis, University of Oxford, 2009.

[24] D. J. Szwer, S. C. Webster, A. M. Steane, and D. M. Lucas, J. Phys. B 44, 025501 (2011).

[25] D. J. Berkeland, J. D. Miller, J. C. Bergquist, W. M. Itano, and D. J. Wineland, Phys. Rev. Lett. 80, 2089 (1998).

[26] J. J. Bollinger, D. J. Heizen, W. M. Itano, S. L. Gilbert, and D. J. Wineland, IEEE Trans. Instrum. Meas. 40, 126 (1991).

[27] K. Saeedi, S. Simmons, J. Z. Salvail, P. Dluhy, H. Riemann, N. V. Abrosimov, P. Becker, H.-J. Pohl, J. J. L. Morton, and M. L. W. Thewalt, Science 342, 830 (2013).

[28] E. Knill, D. Leibfried, R. Reichle, J. Britton, R. B. Blakestad, J. D. Jost, C. Langer, R. Ozeri, S. Seidelin, and D. J. Wineland, Phys. Rev. A 77, 012307 (2008).

[29] D. P. L. Aude Craik, N. M. Linke, T. P. Harty, C. J. Ballance, D. M. Lucas, A. M. Steane, and D. T. C. Allcock, Appl. Phys. B 114, 3 (2014).

[30] M. H. Levitt, Prog. Nucl. Magn. Reson. Spectrosc. 18, 61 (1986).

[31] C. J. Ballance et al. (to be published).

[32] J. Benhelm, G. Kirchmair, C. F. Roos, and R. Blatt, Nat. Phys. 4, 463 (2008).

[33] G. Kirchmair, J. Benhelm, F. Zähringer, R. Gerritsma, C. F. Roos, and R. Blatt, Phys. Rev. A 79, 020304 (2009). 